\title{
L'IRRADIATION DU LAIT AUX ETATS-UNIS
}

\author{
par \\ C. WOLF
}

\section{Généralités}

Depuis 1931, les laits contenant de la vitamine D font, aux Etats-Unis, l'objet d'un commerce important. C'est, en effet, à cette date que le premier lait liquide à vitamine $\mathrm{D}$ fut mis sur le marché. On estimait, en 1936, que la consommation journalière de ces laits dépassait trois millions de quarts, c'est-à-dire près de 2.840.000 litres. Cette consommation a, du reste, considérablement augmenté depuis cette époque.

Le lait à vitamine $\mathrm{D}$ le plus consommé est le lait irradié, liquide ou évaporé, quoique des laits métabolisés, obtenus en alimentant des vaches à l'aide de levures irradiées, ou des laits additionnés d'un concentré de vitamine D soient également vendus en grande quantité.

Très peu d'années après la découverte en 1921 de la vitamine $D$ dans l'huile de foie de morue, un certain nombre de chercheurs trouvèrent que la lumière ultra-violette provenant, soit du soleil, soit de sources artificielles, agissait sur la peau humaine en produisant des substances antirachitiques ayant le même effet physiologique que la vitamine D. En 1924, Alfred F. Hess et le Pr Harry Strennock publièrent presque simultanément, et indépendamment l'un de l'autre, les résultats d'expériences prouvant que des propriétés antirachitiques pouvaient être communiquées à certains aliments par exposition à l'irradiation à l'aide de lumière ultraviolette.

Un brevet pour ce procédé fut accordé en 1928 au Pr STEE NBock (brevet américain no 1.680 .818 ), qui le céda à la "Wisconsin Alumni Research Foundation ». Entre temps, il avait été indiqué à He ss qu'un aliment aussi répandu que le lait, qui est la meilleure source au point de vue alimentaire du calcium et du phosphore qui sont, comme la vitamine $D$, indispensables pour prévenir le rachitisme, serait le produit alimentaire devant le plus logiquement être soumis à I'irradiation. Avec la coopération de G. C. SUPPLEE, des Laboratoires de Recherches de la "Dry Milk Company " Hess commença, en 1925, une série d'études sur les laits à vitamine D qu'il poursuivit jusqu'à sa mort en décembre 1933 et qui furent continuées par la suite par ses collaborateurs et collègues.

Le résultat de ces études fut le lancement sur le marché, en 1927, d'un lait en poudre irradié (Dryeo) et l'obtention, en 1931, 
d'un brevet de préparation d'un lait irradié par SuPPLEe. En 1928, T. F. ZUCKER, de PUniversité Colombia, breveta une méthode d'extraction de l'huile de foie de morue et d'autres produits, de la vitamine antirachitique et, quelques années plus tard, une méthode fut imaginée pour ajouter ces concentrés au lait. Depuis cette époque un assez grand nombre d'autres concentrés de vitamine D ont été préparés pour être utilisés dans la fabrication de laits à vitamine D.

Toutefois, le premier lait liquide à vitamine $\mathrm{D}$ mis sur le marché ne fut ni un lait irradié, ni un lait additionné d'un concentré de vitamine $\mathrm{D}$, mais un lait certifié enrichi naturellement en vitamine D par des méthodes scientifiques d'alimentation du bétail. Des expériences effectuées à la ferme Walker-Gordon, à Plainsboro (New-Jersey) avaient établi que des vaches alimentées avec de la levure ou de l'ergostérol irradiés donnaient des laits beaucoup plus riches en vitamine D que les laits ordinaires qui, quoique contenant un peu de vitamine $D$, n'en possèdent pas suffisamment pour protéger les enfants contre le rachitisme, ce qui, en passant, est, du reste, également le cas pour la plupart des laits maternels qui manquent généralement de vitamine $\mathrm{D}$. Des études ultérieures montrèrent que la levure irradiée était supérieure à l'ergostérol irradié pour la production du lait à vitamine $\mathrm{D}$.

Les essais de laboratoire furent suivis d'essais cliniques sur une centaine d'enfants, essais qui furent couronnés de succès. Aussi, quelques mois plus tard, en 1931, un lait certifié additiónné de vítamine D, était-il vendu à New-York, Boston et Philadelphie.

Du lait liquide pasteurisé irradié fut mis en vente à Détroit vers la fin de 1932 et à peu près à la même époque, du lait additionné d'un concentré de vitamine $\mathrm{D}$ fut également mis sur le marché. Le lait évaporé irradié ne fut mis dans le commerce qu'au début de 1934. Depuis 1932, la vente de nombreux laits liquides et évaporés à vitamine $\mathrm{D}$ a été autorisée par le Comité des Aliments de I' "American Medical Association ".

Nous ne nous occuperons dans la présente étude que de l'irradiation du lait.

\section{Raisons physiologiques de I'irradiation}

La valeur antirachitique du lait irradié est plus souvent indiquée sous le terme de potentiel en vitamine $\mathrm{D}$ qui désigne la valeur de ce lait dans son action thérapeutique ou active contre le rachitisme. Le rachitisme est une maladie constitutionnelle de l'enfance qui rend les os mous et flexibles par suite d'une ossification retardée. Cela peut être du à une déficience de calcium, phosphore, vitamine D ou en lumière solaire, ou peut-être à d'autres troubles du métabolisme. 
Le contrôle prophylactique convenable du rachitisme nécessite un juste équilibre entre le calcium, le phosphore et un facteur antirachitique. Le calcium et le phosphore, éléments minéraux, peuvent être founis par l'alimentation. Le facteur antirachitique, c'est-à-dire la vitamine $\mathrm{D}$, aide à l'assimilation et à la rétention des éléments minéraux fournis par l'alimentation. La présence de ce facteur est limitée dans la nature à de très rares produits alimentaires et notre mode actuel de vie fait qu'il n'est pas suffisamment disponible pour correspondre aux exigences physiologiques. Si on voulait assurer une protection convenable à l'organisme contre le rachitisme par un choix d'aliments riches en vitamine $\mathrm{D}$, il en résulterait des troubles digestifs ou un déséquilibre alimentaire.

\section{Technique de l'irradiation}

On peut définir l'irradiation du lait liquide comme étant "l'exposition de ce lait liquide à des rayons ultra-violets en qualité et en quantité convenables, dans des conditions d'exposition convenablement contrôlées, pour que le produit obtenu ait des propriétés mesurables et cliniquement démontrables pour Ia prévention du rachitisme infantile " (1).

La vitamine $\mathrm{D}$ peut être obtenue comme constituant naturel et partiel d'un assez grand nombre de produits biologiques ou par activation de certains composés existant naturellement, ou comme ils existent dans les produits après séparation, concentration ou purification.

Le lait contient à la fois de la vitamine $\mathrm{D}$ à l'état de constituant naturel et sous la forme de constituants qui, exposés à la radiation, sont convertis en produits contenant de la vitamine. La teneur naturelle du lait en vitamine $D$ est en apparence variable et dépend d'un certain nombre de facteurs, principalement du type de nourriture consommée et de l'exposition de la vache au soleil; mais dans les conditions où le lait est produit pratiquement, ces variations n'ont probablement aucune importance.

Le tableau ci-après donne, d'après certains expérimentateurs, une idée de la variation de la teneur du lait en vitamine $\mathrm{D}$ naturelle.

La teneur du lait en vitamine $D$ est généralement fixée par essais biologiques ("line test " ou "bone ash ") sur des rats. Il ne semble pas qu'on ait trouvé jusqu'à présent un procédé chimique ou photochimique pour la détermination de la teneur en vitamine susceptible de remplacer la méthode biologique longue et coûteuse. Toutes celles qui ont été proposées n'ont pas été trouvées suffi-

(1) G. C. Supplee, "Committee on definitions of irradiation of milk». Hôtel Roosevelt, New-York, 22 juillet 1935. 
Potentiel en Unités U.S.P. par quart ( $\left(\begin{array}{lll}0 & 1.946\end{array}\right)$ de lait

\begin{tabular}{|c|c|c|c|}
\hline Hiver & Eté & & \\
\hline 3,1 & 27,0 & Holstein & Bechdell et Collaborateurs \\
\hline 4,9 & 43,8 & Guernsey & Bedchdell et Collaborateurs \\
\hline 7,3 & 16,0 & Holstein & Krauss et Collaborateurs \\
\hline 21,0 & 43,0 & Holstein & Steenbock et Collaborateurs \\
\hline 23,0 & 43,0 & Troupeau & 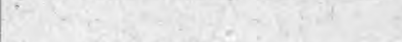 \\
\hline & & mélangé & Russell et Collaborateurs \\
\hline Ration d'hiver: & Ration d'été : & & \\
\hline & (à l'intérieur) & & \\
\hline 57,4 & $\begin{array}{c}37,5 \\
\text { (en plein air) }\end{array}$ & Shorthorn & \\
\hline
\end{tabular}

N. B. - Les Unités "United States Pharmacopocia "sont équivalentes à l'U. I., c'est-à-dire à 0,025 microgrammes de vitamine $\mathrm{D}$ cristallisée.

samment précises pour répondre aux exigences des méthodes de contrôle nécessaire en matière d'hygiène publique (1).

Le stérol qui a probablement la plus grande importance comme provitamine existant dans le lait est le 7 - déhydrocholestérol. La matière grasse du lait contient la plus grande concentration de stérol, environ $0,3 \%(2)$. La teneur en cholestérol du lait varie entre 10 et 15 milligrammes par 100 millilitres et celle du lait écrémé de 1 mgr. 5 à 3 mgr. 5 par 100 millilitres. Une partie du cholestérol du lait (environ $18 \%$ ) est associé en combinaison prosthétique avec la lactalbumine. On pense que c'est à cette combinaison qu'est due l'efficacité des substances à vitamine $\mathrm{D}$ du lait. La transformation du 7-déhydrocholestérol en 7-déhydrocholestérol activé (vitamine $\mathrm{D}^{3}$ ) paraît s'effectuer d'après le schéma ci-après :

(1) Note de la Rédation : On consultera avec profit "Dosage spectrophotométrique du calciférol en solution organique complexe ( $M$. Vaeher, $M^{\text {lles }} Y$. Lortie et $H$. Colson). Bull. Soc. Chimie biologiqu', 1944, 26, 206.

(2) S. Ansbacher et G. C. Supplee. The cholesterol content and the antirachitic activation of milk constituents. Journal Biol. Chem., 105, 391, 1934. 


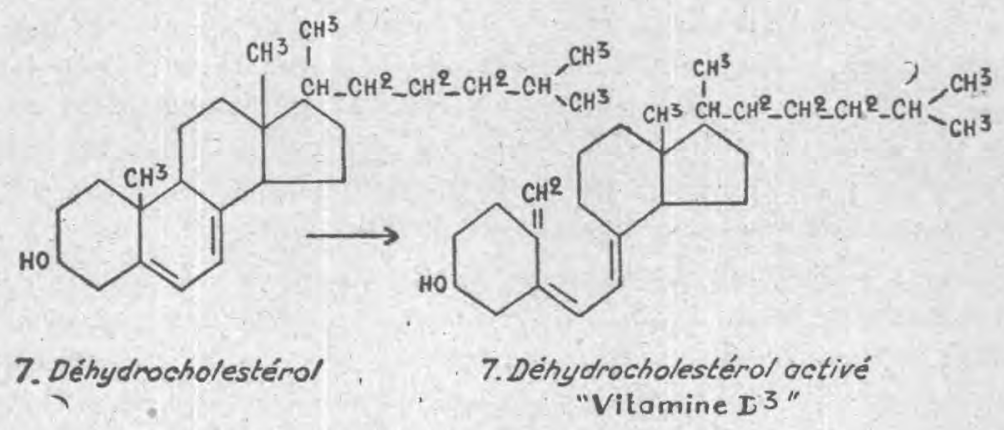

\section{Sources artificielles de radiation ultra-violette}

La lumière $d u$ soleil n'est pas une source de radiation ultraviolette sur laquelle on puisse compter dans nos régions septentrionales, en raison de sa variation pendant les diverses périodes de l'année et de l'intervention de facteurs naturels tels que la variation de la distance de la terre au soleil, la proportion de vapeur d'eau dans l'atmosphère, les poussières ou la brume de cette atmosphère, la distance zénithale du soleil et l'altitude au-dessus du niveau de la mer.

Aussi, emploie-t-on généralement trois sources artificielles de radiation pour l'irradiation du lait : la lampe à are de carbone et les lampes à are en quartz à vapeur de mercure chaude et à vapeur de mercure froide.

\section{Lampes à arc de carbone}

Les électrodes de l'are de carbone utilisé pour l'irradiation du lait consistent en tubes de carbone dont l'intérieur est imprégné de métal, quelquefois même de plusieurs métaux, ce qui les fait, dans ce cas, dénommer carbones polymétalliques. La eomposition de la radiation peut varier qualitativement et quantitativement dans certaines limites quand on modifie la composition de l'intérieur métallique et le courant électrique. A l'exception de la bande à 3.890 Angstroms $(\AA)$, les vapeurs des électrodes de carbone pur sont relativement non lumineuses. Un arc lumineux est créé quand les carbones sont imprégnés de divers métaux; la présence du strontium crée une flamme rouge et celle du cobalt une flamme 
bleue. La plus grande partie de la radiation provient essentiellement du jeu de l'are entre les électrodes. L'intensité de la radiation ayant un effet antirachitique varie considérablement suivant les divers carbones utilisés et suivant les conditions. Il y a en outre, une différence entre les proportions relatives de radiation dans des zones délimitées entre 2.300 et 3.100 Angstroms.

L'arc de carbone peut fonctionner à l'aide de courant continu ou alternatif. Dans une des dispositions utilisées, l'are est produit quand les carbones sont dans une position verticale. Les carbones supérieurs des ares ayant une émission de radiation intense sont fréquemment de diamètre plus important, afin de maintenir une vitesse de combustion égale (oxydation des électrodes) des carbones supérieurs et inférieurs, les carbones supérieurs baignant dans la flamme de l'arc. Pour la même raison et pour augmenter la conductibilité électrique, les carbonès sont recouverts de cuivre. Des ares produits quand les carbones sont en position horizontale ou angulaire peuvent également, par une adaptation convenable, être utilisés pour l'irradiation du lait. Un moteur électrique à marche réversible contrôle mécaniquement les mouvements, dépendant, du reste, tous les uns des autres, tels que la mise en marche des arcs, l'intervalle entre les carbones, la longueur de la flamme, et l'intensité du courant et de la radiation. Une ventilation convenable doit enlever les produits gazeux de la combustion de l'arc. Cette ventilation doit être régulière et continue pour ne pas gêner le bon fonctionnement de l'are par des courants d'air croisés ou des remous d'air.

L'are de earbone a été adopté pour l'irradiation du lait quand ce dernier coule sur une surface verticale telle qu'un réfrigérant à Iait, à l'intérieur d'un cylindre ou d'un tronc de cône et comme un film cylindrique sans support autour de l'are.

Un appareil comportant essentiellement un réfrigérant à surface plane (I) permet l'exposition à un arc de carbone du lait en couche mince sur une face du réfrigérant d'une surface rectangulaire d'environ $3 \mathrm{~m}^{2} 25$. La distance perpendiculaire de l'are de earbone au centre de la surface rectangulaire exposée est de $0 \mathrm{~m}$. 50. La radiation émanant de l'hémisphère de l'are la plus rapprochée de la surface exposée est une radiation directe et celle de l'hémisphère plus éloignée est redirigée en partie sur le lait par un réflecteur qui renvoie environ $60 \%$ ou plus de da radiation effective. Dans cet appareil, le lait introduit par un dispositif d'alimentation constante arrive sur la surface d'exposition par. un distributeur 
linéaire. Le conduit de ventilation et les eanalisations électriques sont placés au-dessus de la lampe.

Le type conique d'irradiateur consiste en une lampe à arc dè carbone suspendue à l'intérieur d'un trone de cône renversé. La lampe est suspendue au centre de l'irradiateur. La radiation émanant de tous les points de l'hémisphère horizontal autour de l'arc sert à l'irradiation du lait. La ventilation est assurée par un ventilateur aspirant placé au-dessus de l'irradiateur. La distribution et le mouvement, de l'air à travers l'appareil sont rendus possibles par la présence d'un tamis placé à la base du trone de cône. Le lait est introduit à l'aide d'une pompe à alimentation constante par une auge de distribution cylindrique sur la surface intérieure de l'irradiateur et à son sommet. Le lait tombe du distributeur sur la surface intérieure dù trone de cône où il est exposé en couche mince à la radiation de l'arc. Le lait est rassemblé et sort de l'appareil par une auge placée à la partie inférieure de l'appareil.

Un type plus récent d'appareil cylindrique à arc de carbone est caractérisé par la plus grande intensité de la radiation émanant de l'arc provenant d'une lampe à arc à six carbones. Le lait coule d'une ange distributrice à déversoir à la partie supérieure de l'irradiateur. Le lait est exposé à l'irradiation quand il s'écoule en tombant le long de la surface intérieure de l'appareil. Il est ensuite rassemblé dans une auge placée à la base de l'appareil d'où il est enlevé. La grande capacité de l'appareil est due à la plus grande intensité de radiation émanant de la lampe et à une utilisation plus avantageuse de cette radiation.

Le matériel utilisé pour l'irradiation d'une couche de lait sans support est appelé le "Double Bubble Irradiator " (1) (irradiateur à double cloche). Le film sans support est produit en expulsant le lait sous pression par un orifice annulaire étroit. La couche mince forme les parois d'un cylindre creux de $0 \mathrm{~m} .21$ de diamètre et de $0 \mathrm{~m} .33$ de hauteur. Cette couche mince cylindrique coule à très grande vitesse. L'are est placé au centre du film cylindrique de lait. Le lait est protégé contre les gaz volatils et les résidus de l'are par l'interposition d'un second film cylindrique d'eau sans support entre l'are et le film du lait. Ce cylindre d'eau a un diamètre de $0 \mathrm{~m} .09$. Un ventilateur enlève les vapeurs de l'arc à travers ce cylindre d'eau. L'eau emporte les résidus solides de l'arc vers un tamis d'où ils sont enlevés. Le courant est fourni aux carbones à travers des porte-carbones qui entourent les carbones à l'intérieur du film d'eau.

(1) Cherry Burrell. S. Corp., Chicago, I1l. 


\section{Lampes en quartz à arc à vapeur de mercure}

Les-lampes à are à vapeur de mercure sont très utilisées comme source d'énergie radiante. Le brûleur, ou élément d'ar'e, est établi sous plusieurs formes. Dans une forme ancienne, le brûleur était construit entièrement en quartz transparent fondu et contenait des cuvettes de mercure liquide agissant comme électrodes en fournissant de la vapeur de mercure à l'arc. Dans une forme plus récente, il est de forme tubulaire, construit entièrement en quartz fond $u$ transparent et ne contient pas de mercure visible, la décharge de l'aro se faisant entre deux électrodes activées solides. Le brûleur s'allume automatiquement quand le commutateur de force est fermé. Il est construit en tubes plus longs et donne des unités plus puissantes qu'avec les anciens modèles et son utilisation a également permis de simplifier le circuit électrique. Dans un autre type de lampe à vapeur de mercure, l'anode est en tungstène solide et la cathode à cuvette de mercure. Ces arcs de mercure à quartz sont dénommés arcs chauds à haute pression. Au début, le brûleur est froid, de sorte que la pression de la vapeur de mereure à l'intérieur est basse, le voltage du brûleur est bas et le courant du brûleur élevé. Quand la température de l'arc s'élève, la pression de vapeur et le voltage augmentent et l'ampérage diminue.

Les caractéristiques des arcs à vapeur de mercure dans lesquels il y a des cuvettes de mercure libre sont influencées par les températures extérieures. Les lampes n'ayant pas de cuvettes de mercure sont moins influencées par les changements de température. Les fluctuations du voltage fourni et les pertes dans la transmissibilité de l'enveloppe de quartz fondu qui a ugmentent avec l'âge et l'usage sont des facteurs influençant le rendement dè la lumière. Il en résulte que les applications de l'arc de mercure à quartz à l'irradiation du lait ont été nécessairement accompagnées par l'emploi de cellules photoélectriques ou de l'emploi de tests photochimiques mesurant directement, ou indirectement par interprétation, l'intensité de certaines radiations. La cellule photoélectrique a été adaptée au contrôle de l'arrivée du courant à l'arc de façon à corriger les fluctuations de l'émission de lumière dues aux modifications de voltage et aux modifications de la transparence $d u$ quartz. Il est ainsi possible de maintenir pratiquement constante l'intensité des radiations ultraviolettes sur la surface du lait pendant le fonctionnement utile du brûleur.

La lampe en quartz à arc à vapeur de mercure chaude est généralement employée pour l'irradiation du lait de deux façons générales : $1^{\circ}$ en dirigeant la radiation sur le lait coulant sur une surface verticale ou presque verticale; $2^{\circ}$ en exposant le lait se 
déplaçant à l'intérieur d'un cylindre fermé à la radiation d'un aro placé à l'intérieur du cylindre.

Dans un appareil du premier type, le lait arrive dans une cuve dont le niveau est réglé par un robinet à flotteur. Il passe ensuite par des auges distributrices sur des surfaces légèrement inclinées sur la vertieale sur lesquelles il est exposé à la radiation émanant d'un brûleur placé au centre entre deux surfaces rectangulaires. La cellule photoélectrique utilisée pour maintenir l'émission du brôleur est placée à une courte distance an-dessus de l'arc. Ces appareils existent en plusieurs dimensions avec des capacités allant de 570 à 3.850 kilográmmes de lait par heure. Dans les grandes unités, des capacités plus importantes sont obtenues par l'adaptation de brûleurs à mercure multiples. Comme il s'écoule un certain temps avant que l'émission de radiation du brûleur en quartz à vapeur de mercure convienne à l'irradiation du lait, il est nécessaire que les brûleurs soient mis en route dans une chambre de chauffage d'où ils sont ensuite transférés à la chambre d'irradiation proprement dite. Les brûleurs étant enfermés, il n'existe pas de produits de combustion dans ces lampes. La chaleur produite est enlevée par ventilation naturelle ou par des chambres à refroidissement par eau. Dans certains appareils, le brûleur à courant continu est logé dans un tube de quartz cylindrique horizontal. Le lait passe autour de ce tube dans une direction longitudinale d'une extrêmité à l'autre. La marche du courant étant dirigée sur un eertain nombre de chicanes, le lait est done obligé de passer à travers le petit orifice formé entre les chicanes et le cylindre de quartz. Dans ces orifices, le lait est exposé en couche mince à la radiation transmise. Le brûleur, le cylindre de quartz et les chicanes sont logés dans une chambre métallique. Ce lait est ainsi irradié en chambre close et n'est pas exposé à l'air. Dans un autre type d'appareil, le lait passe sur la surface intérieure d'un petit eylindre vertical dans le centre duquel est placé un arc. Une efficacité plus grande de l'irradiation est assurée par l'interposition d'anneaux placés à diverses hauteurs du cylindre et par l'agitation du lait.

Le tube à déeharge à haut voltage, appelé habituellement tube de quartz froid, a aussi été adapté à l'irradiation du lait. Le brûleur consiste essentiellement en un tube de quartz fondu d'environ 9 millimètres de diamètre, dans lequel le vide a été très poussé et l'air remplacé par une atmosphère de gaz rares (xénon, crypton et argon) et quelques gouttes de mercure. Extérieurement, ces brûleurs ressemblent beaucoup à ceux au néon utilisés- pour la publicité. Ces lampes fonctionnent avec une très faible consommation de courant (environ 15 à 100 milliampères), mais à très fort voltage. Une des caractéristiques de la décharge à haut voltage 
est l'absence relative de la chaleur de la radiation. La plus grande partie de cette radiation tombe dans la zone regardée comme antirachitique ; mais son intensité est relativement faible. Il est donc nécessaire d'employer un grand nombre de tubes à proximité immédiate du lait.

Il est évident que le courant du lait exposé à la radiation doit être constant. La continuité et la simultanéité du travail des pompes ou des auges d'alimentation avec les arcs ultra-violets est assurée par l'utilisation d'appareils enregistreurs.

\section{Réglage de l'intensité de l'irradiation}

Pour obtenir un produit de potentiel antirachitique uniforme et satisfaisant, il est nécessaire que l'irradiation du lait soit opérée par ùne radiation uniforme et complète de la source ultra-violette. L'intensité de la radiation d'un are ultra-violet est surtout commandée par le courant appliqué à l'are. Il est done indispensable que les caractéristiques électriques de l'arc, ou de la radiation qu'il produit, soient visibles ou contrôlées. L'intensité de la radiation des arcs ultra-violets peut être maintenue et notée, soit par la mesure de la puissánce fournie à l'are, soit par celle de la radiation émise par l'are. Alors que dans le cas de l'arc à carbones, les appareils habituels de mesure du voltage, de l'ampérage et du wattage suffisent, pour l'arc à vapeur de mercure la mesure des caractéristiques du courant n'est pas suffisante pour donner une idée de l'intensité de la radiation. L'emploi de la cellule photoélectrique permet d'y parer.

\section{Composition. du lait}

La vitamine D étant surtout associée avec la fraction grasse du lait, il paraît évident que la teneur en matière grasse du lait a une influence sur la proportion de vitamine D formée par l'irradiation. Il a été prouvé expérimentalement que pour des laits irradiés d'une façon comparable, la teneur en vitamine D augmente quand la teneur en matière grasse du lait augmente de celle du lait écrémé jusqu'à $7,2 \%$, quoique l'augmentation en vitamine D puisse ne pas être proportionnelle à la teneur en matière grasse. Les laits contenant relativement peu ou pas de matière grasse peuvent acquérir une quantité assez importante de vitamine $D$ par l'irradiation; mais le degré de potentiel définitivement obtenu n'est pas atteint aussi rapidement que dans les laits contenant une forte proportion de matière grasse. Le potentiel antirachitique aequis par les laits pauvres en matière grasse peut être attribué en partie à la présence de substances activables combinées avec la fraction protéine, telle que l'albumine du lait. 
La crème peut être exposée à la radiation dans des conditions analogues à celles employées pour l'irradiation du lait. Le potentiel antirachitique du lait reconstitué préparé avec de la crème irradiée et du lait écrémé non irradié est analogue à celui du lait complet irradié normal. Geci indique que pour toutes les applications pratiques la principale partie du précurseur est associée avec la matière grasse du lait. Il a été indiqué que, dans des conditions expérimentales similaires, le lait fluide acquiert un potentiel antirachitique plus important que le lait évaporé, étant entendu que la comparaison est faite après que le lait évaporé a été reconstitué à la composition du lait normal.

Des variations dans la qualité de la ration normale (alimentation d'hiver ou pâturage ; peu de protéine ou beaucoup ; ensilage A.I.V. ou foin de luzerne) ont de l'influence sur la teneur naturelle en vitamine D du lait. Quand les laits sont irradiés dans des conditions comparables, l'activité produite dans le lait naturellement pauvre en vitamine $\mathrm{D}$ ne semble pas différente de celle du lait naturellement riche en vitamine D. Le dernier type peut posséder un potentiel en vitamine D plus élevé à la suite de l'irradiation que le premier ; mais il semble probable que ceci peut être attribué au potentiel originel plus élevé du lait non irradié.

\section{Caractéristiques de la couche de lait}

Le lait, même en couches minces, est relativement impénétrable aux rayons ultra-violets antirachitiques. Les radiations de $2.850 \mathrm{~A}$ ou moins sont absorbées dans la proportion d'environ 60 à $80 \%$ par les premiers $0 \mathrm{~mm} .02$ de la couche de lait; $95 \%$ ou plus sont. absorbés par les premiers $0 \mathrm{~mm}$. 11. Il a été démontré que seulement $4 \%$ de la radiation ultra-violette d'une longueur d'onde de $2.800 \AA$ traversent la surface arrière d'une couche épaisse de $0 \mathrm{~mm}$. 2. L'opacité du lait à la radiation de $3.000 \AA$ et moins est due prineipalement à la matière grasse et à la caséine, quoique les constituants solubles du lait absorbent cette radiation dans une proportion importante. Le lait est moins opaque à la radiation de longueurs d'onde supérieures à $3.000 \AA \bar{A}$ qu'à la radiation de longueurs d'onde inférieures à $3.000 \AA$. La matière grasse, la caséine et l'albumine transmettent plus facilement la radiation des ondes plus longues.

Puisque la pénétration de la radiation antirachitique active dans la couche de lait n'est pas très grande, l'état physique de cette couche est important dans la détermination de l'efficacité de la radiation, mesurée en termes de potentiel antirachitique et appliquée dans des conditions constantes. 


\section{Caractéristique de la radiation}

Deux caractéristiques doivent être étudiées quand on choisit un are pour l'irradiation du lait : l'intensité de la radiation active au point de vue antirachitique et la qualité du spectre.

L'efficacité de la radiation émanant d'une source centrale sur le potentiel antirachitique du lait dépend en partie de l'intensité de l'émission déterminée par la distance des différentes portions de la couche à la source de radiation. Il semble préférable d'utiliser des couches de lait ayant une capacité plus grande que 744 kilogrammes par mètre linéaire et par heure pour maintenir les standards de potentiel dans des conditions opératoires difficiles.

Il est évident que la modification de l'intensité de la radiation d'un arc modifie le potentiel antirachitique du lait. La radiation s'applique le mieux quand l'are est à une distance de $0 \mathrm{~m} .15$ de la couche de lait pour la lampe à arc à vapeur de mercure et de $0 \mathrm{~m} .30$ à $0 \mathrm{~m} .45$ pour la lampe à are de earbone. Le potentiel antirachitique maximum est donné au, lait par un apport d'énergie de 300 joules par gramme de lait.

\section{Autres effets de la radiation}

L'irradiation ne diminue pas de façon sensible la proportion de vitamine $\mathrm{A}$ ou de carotène dans le lait fluide et dans le lait évaporé. Il ne semble pas que les vitamines $B$ et $G$ soient affectées par les expositions de quelques secondes aux rayons ultra-violets utilisées dans l'irradiation du lait. On a pu observer une légère diminution du potentiel en vitamine $\mathrm{C}$ par l'irradiation; mais il est possible que cette diminution soit plutôt attribuable à l'aération, qui a lieu pendant l'opération qu'à l'irradiation elle-même. Dans les conditions normales de l'irradiation, des réductions assez importantes de la teneur en bactéries du lait ( 7 à $37 \%$ ) ont lieu ; mais ces réductions ne sont pas suffisantes au point de vue pratique pour dire que l'irradiation améliore la qualité du lait au point de vue hygiénique. On a aussi constaté que l'irradiation du lait produit un ralentissement net du développement de l'acidité.

Il peut résulter d'une application défectueuse de l'irradiation une saveur dénommée "activée » qui, à son début, peut être qualifiée de "brûlée », de "plume brûlée ", de "protéine brûlée», ou même dans certains cas, de "saveur de champignon ". II semble que certaines longueurs d'onde (1) doivent êtres exclues pour l'irradiation si on veut éviter la saveur « activée ». En outre, l'utilisation de réoipients en verres colorés, en verre filtrant ou recouverts de

(1) Les radiations de longueurs d'onde de 3.100 ì 3.800 A produisent plus de saveru que celles de 3.800 à $7.000 \AA$. 
cellophanes colorées évite le développement de cette saveur "activée ".

L'irradiation normale du lait ne parait pas produire de modification sensible de la composition ou de la digestibilité du lait.

\section{Méthodes officielles utilisées pour les essais biologiques de la vitamine $D$ du lait}

Ainsi qu'il a déjà été indiqué, les essais biologiques de la vitamine $\mathrm{D}$ du lait sont effectués sur des rats. Ces rats nés de rats nourris avec une ration pauvre en potentiel antirachitique sont élevés par leur mère jusqu'à ce qu'ils atteignant un poids approximatif de 40 à 60 grammes à un âge de 21 à 28 jours. A ee moment, ils sont soumis à une alimentation produisant le rachitisme (période de déplétion) dans un local où la lumière solaire directe ou réfléchie ne peut pénétrer et à une température variant entre $22^{\circ} 2$ et $26^{\circ} 6$. Les rats sont rassemblés par groupes d'essai d'au moins sept et il ne doit pas y avoir plus de trois rats de la même portée dans un groupe d'essai à moins qu'il n'y en ait un nombre égal dans le groupe-témoin. Ce groupe-témoin reçoit de l'huile de foie de morue standard (réf. U.S.P.) qui servira de mesure comparative pour l'essai de la vitamine D du lait. Pendant la période d'essai, ce groupe recevra, outre l'alimentation rachitogénique, de la poudre de lait écrémé (obtenue par extraction par l'éther) égale en poids à l'extrait sec dégraissé contenu dans le lait donné au groupe d'essai. Ce supplément doit être donné journellement pendant toute la période pendant laquelle le groupe d'essai reçoit du lait.

Un rat convient à l'essai après une période de déplétion de dix-huit à vingt-einq jours et s'il manifeste nettement du rachitisme. La présence du rachitisme peut être établie par l'examen d'un os ou d'une patte d'un animal d'une portée par le "line test" décrit plus loin ou par l'examen aux rayons $\mathrm{X}$.

Les rats convenant à l'essai sont pesés et isolés dans des cages individuelles à fond à claire-voie et reçoivent l'alimentation rachitogénique et de l'eau distillée $a d$ lib. La période d'essai dure sept jours. Pendant les six premiers jours de la période d'essai, chaque rat des groupes d'essai reçoit journellement la dose caleulée de lait à vitamine $\mathrm{D}$ et chaque rat des groupes-témoins une dose d'huile standard. Les rats sont tués à la fin de l'essai, et un ou plusieurs os des pattes sont examinés en vue de la guérison des métaphyses rachitiques suivant la méthode dite "line test".

L'huile standard est diluée préalablement avec une huile végétale comestible exempte de vitamines A et D. On ne doit pas donner plus de $0 \mathrm{~cm}^{3} 1$ de l'huile standard diluée par jour. 
On effectue l'examen dénommé "line teste " sur l'extrémité proximale du tibia ou l'extrémité distale du radius ou du cubitus. L'extrêmité de l'os choisi enlevé de l'animal est débarrassée du tissu adhérent. Une section longitudinale médiane est pratiquée à l'extrémité de l'os afin d'obtenir une surface plane à travers la jonction de l'épiphyse et de la diaphyse. Les deux sections de l'os rincées dans l'eau distillée sont plongées pendant une minute dans une solution aqueuse à $2 \%$ de nitrate d'argent, puis rincées dans l'eau distillée, et les surfaces sectionnées sont exposées ensuite à la lumière du jour ou à une autre source de lumière actinique jusqu'à ce que les surfaces calcifiées soient nettement colorées. La preuve de la congestion dans les métaphyses rachitiques doit être nettement distinguée des sels de calcium colorés par l'argent, ear les eritères principaux de la guérison sont le développement de la ligne à la zone de calcification provisoire. On peut aussi, après sectionnement des os, les placer dans du formol à $10 \%$ ou de l'alcool à $95 \%$ pendant trois à quatre heures. Après rinçage dans l'eau distillée, on colore comme précédemment.

Les résultats de l'étendue et du degré de calcification des métaphyses rachitiques de chaque coupe, doivent être notés immédiatement après coloration. Des valeurs numériques doivent être données au développement et au degré de calcification des métaphyses rachitiques des os examinés, de façon à ce qu'il soit possible de donner une moyenne au groupe. Les expérimentateurs utilisant l'extrêmité distale des radius et eubitus doivent utiliser la méthode déerite par DYer (Quart. J. Pharm. and Pharmocol., 4, 503, 1931) pour l'évaluation numérique. Si on utilise le tibia, on utilise la méthode décrite par Bulls ( $J$. Biol. Chem., 90, 619, 1931). Les poids de chaque animal sont notés le jour du début de l'essai ainsi que le septième jour, et on note également la quantité d'aliment rachitogénique consommée par rat pendant la période d'essai.

Pour déterminer le potentiel en vitamine $\mathrm{D}$ du lait, on se base sur les résultats moyens des groupes en ce qui concerne la guérison de la métaphyse rachitique, étant entendu qu'une telle moyenne d'un groupe est déterminée par des chiffres provenant de rats qui, individuellement, ont une étendue et un degré de calcification égal ou supérieur à un état décrit comme preuve macroscopique positive de la calcifieation, mais moindre qu'une étendue et un degré de calcification décrits comme guérison complète. Les chiffres d'un groupe-témoin ne seront considérés comme valables pour établir le potentiel en vitamine D du lait que lorsque les deux-tiers du groupe ou plus, mais pas moins de 7 rats, montrent individuellement la preuve macroscopique de guérison, mais pas de guérison complète. Les chiffres d'un groupe d'essai ne sont considérés 
comme valables pour établir qu'un lait soumis à l'essai est conforme au potentiel noté en termes d'unités U.S.P. que lorsque pas moins de sept rats montreront individuellement la preuve macroscopique de la guérison. Les chiffres d'un rat ne sont considérés comme valables pour établir la moyenne d'un groupe qu'à la condition que le poids du rat à la fin de l'essai soit égal ou dépasse le poids du rat au début de la période d'essai et que le rat ait consommé 28 grammes ou plus de l'aliment rachitogénique pendant la période d'essai et à la condition que le rat ait consommé chaque dose de lait prescrite dans les vingt-quatre heures suivant le moment où ce lait lui a été donné.

Le potentiel en vitamine D du lait est ensuite calculé comme suit :

$$
\mathrm{Y} \times \frac{946}{\mathrm{X}}=\text { unités U.S.P. par quart }\left(946 \mathrm{~cm}^{3}\right)
$$

dans laquelle $Y=$ unités U.S.P. de vitamine D (dose totale) nécessaire pour produire une ligne de calcification étroite dans les groupes d'essai. X est le nombre de centimètres cubes de lait (dose totale) nécessaire pour produire dans le groupe d'essai un degré de calcifieation égal ou supérieur à celui produit dans le groupe-témoin.

Dans une autre méthode, dénommée "bone ash », le degré de calcification est déterminé par le pourcentage de cendre d'os. Cette méthode est surtout utilisée dans les recherches biologiques expérimentales.

\section{Réglementation de la préparation et de la vente du lait irradié}

Cette réglementation, aussi bien pour le lait vendu en vrac que pour celui vendu en bouteilles, varie aux Etats-Unis d'Etat à Etat et même de ville à ville. Dans certaines villes, il est exigé que le lait irradié soit contrôlé mensuellement pour son potentiel en vitamine D; dans d'autres villes, six fois par an, et, enfin, dans beaucoup de villes, de trois à six fois par an. Ces essais biologiques ont lieu d'après les méthodes indiquées au cours de cette étude. Toutefois, quelques Etats, comme le Connecticut, NewYork, le Kentucky, la Virginie et le Wisconsin, ont des règlementations particulières relatives à la distribution et à la vente des laits à vitamine $\mathrm{D}$ qui exigent entre deux à quatre essais biològiques par an. La "Wisconsin Alumni Research Foundation" exige de ses licenciés au moins trois essais biologiques par an, et que tous les laits soumis à l'irradiation soient pasteurisés. Elle n'autorise pas l'utilisation de l'irradiation sur le lait cru. 
Il ressort d'essais exécutés dans cet établissement par KRIEGER et ScotT (Food Research, mai-juin 1938, vol. III, no 3 ) que la teneur en vitamine $D$ du lait évaporé, irradié, ne diminue pas, ou presque pas, quand ce lait est conservé pendant deux ou trois ans dans des locaux dont la température ne varie que modérément. Pendant la première année de conservation, il ne se produit aucune perte.

\section{Valeur antirachitique du lait irradié dans l'alimentation infantile}

De nombreuses études ont démontré la valeur antirachitique du lait fluide et évaporé dans la prévention du développement du rachitisme. Nous ne pouvons citer que quelques-unes de ces études.

Dans un essai de quatre mois et demi, sur dix-neuf enfants blancs (d'âge moyen de deux mois) avec du lait évaporé irradié contenant 125 unités U.S.P. de vitamine D par boîte de 0 1. 43 et de lait complet fluide irradié contenant 140 unités U.S.P. par quart ( 0 1. 946), on ne constata par examen roentgénographique aucune preuve de développement de rachitisme. Chez un enfant atteint de rachitisme au début de l'essai, la guérison commença au bout de treize semaines et fut complète en dix-huit.

Dans un autre essai, sur vingt-trois enfants nègres alimentés avec du lait évaporé irradié contenant 125 unités U.S.P. de vitamine D par boîte de 0 1. 43, ce lait a prouvé être un agent convenable pour la prévention du rachitisme chez les enfants nègres et avoir une valeur considérable pour la guérison du rachitisme chez les mêmes enfants.

Une étude entreprise sur vingt-deux enfants normaux âgés à l'origine de une à quinze semaines et d'origines ethniques différentes des classes pauvres et moyennes alimentés avec du lait évaporé irradié pendant une période d'un an, montre que ce lait a évité le rachitisme à neuf sur dix des enfants.

Le lait enrichi en vitamine D par irradiation est bien accepté par tous les enfants.

Le fait que les enfants vivent sous un climat ensoleillé ne suffit pas dans les conditions actuelles de la vie pour les préserver du rachitisme.

\section{BIBLIOGRAPHIE}

[1] G. C. Supplee et M. J. Dorcas. Lait irradié : caractéristiques de la couche de lait s'écoulant pour une efficacité optimum de l'activation antirachitique. Journal of Dairy Science, août 1934, vol. XVII, no 8.

[2] M. Rapoport, J. Stokes et D. V. Whipple. Valeur antirachitique du 
lait évaporé irra d ié pour les jeunes enfants. The Journal of Pediatrics, St-Louis, vol. 6, n॰ 6, p. 799, juin 1935.

[3] R. A. Strong, E. F. NAef et I. M. Harper. Propriétés antirachitiques du lait évaporé irradié administré à de jeunes enfants normaux dans des conditions familiales. The Journal of Pediatrics, St-Louis, vol. VII, no 1, p. 21, juillet 1935.

[4] G. C. Supplee et H. C. Rentschler. Valeur du compteur-enregistreur de radiation ultra-violette dans l'irradiation du lait. Médical Record, 18 septembre et 2 octobre 1935 .

[5] T. G. H. Drake, F. F. Tisdale et A. Brown. Nouvelles observations sur l'effet antirachitique du lait frais irradié. The Canadian Medical Association Journal, 34, 279-281, 1936.

[6] M. Rapoport et J. Stokes. Valeur antirachitique du lait évaporé irradié et du lait eomplet fluide irradié pour les jeunes enfants The Journal of Pediatrics, St-Louis, vol. VIII, no 2, p. 154, février 1936.

[7] T. G. H. Drake, F. F. Tisdall et A. Brown. Le lait évaporé irradié dans la prévention du raehitisme. The Journal of Pediatries, StLouis, vol. VIII, $\mathrm{n}^{\circ} 2$, p. 161 , février 1936 .

[8] J. A. Tobey. Les laits à vitamine D. The American Jaurnal of Nursing, vol. XXXVI, $n^{\circ} 6$, juin 1936 .

[9] K. G. Weckel, H. C. Jackson, R. Haman et H. Stennbock. Lait. Effet de différentes sources d'énergie radiante sur la saveur et le potentiel antirachitique. Industrial and Engineering Chemistry, vol. XXVIII, p. 653, juin 1936.

[10] R. W. Haman et H. Steenbock. Equivalents en énergie des unités de vitamine D. Industrial and Engineering Chemistry, vol. VIII, p. 291,15 juillet 1936 .

[11] H. H. Beck et K. G. WeckeL. Caractéristiques de la couche de lait. Effet sur la réaction du lait fluide à la réaction ultra-violette. Industrial and Engineering Chemistry, vol. XXVIII, p. 1251, novembre 1936.

[12] H. T. Sсотт. La lumière et son application à l'irradiation des aliments. Archives of Physical Therapy, X-Ray, Radium, mars 1937, vol. XVIII, p. 154-161.

[13] C. U. Moore, J. L. Brodie, A. J. Thornton, A. M. Lesem et O. B. ConduA. Insuecès d'un soleil abondant pour la protection eontre le rachitisme. The American Journal of Diseases of Children, décembre 1937, vol. LIV, p. 1227-1238 (réimpression avec additions au texte original).

[14] F. O. Tonney et P. P. Somers. Technique revue et eomplète de l'analyse de la lumière solaire actinique. The American. Journal of Hygiene, vol. XXVII, n० 2, 370-379, mars 1938.

[15] W. H. Clark. Valeur du lait irradié dans l'alimentation infantile. Archives of Pediatrics, New-York., 55, 178-184, mars 1938.

[16] C. H. Krieger et H. T, Scotr. Stabilité de la vitamine D dans le lait évaporé irradié. Food Research, mai-juin 1938, vol. III, $\mathrm{n}^{\circ} 3$.

[17] B. O'Brien, H. D. McEwen et K. Morgargidge. Le lait irradié. 
Le potentiel en vitamine $D$ est fonction de l'apport d'énergie. Industrial and Engineering Chemistry, vol. XXX, p. 839, juillet 1938 .

[18] H. T. ScotT et Collaborateurs. Méthodes officielles pour les essais biologiques de la vitamine D du lait. Year Book, 1938-1939. Publié par "The American Public Health Association" 1790, Broadway, New-York, N:-Y.

[19] K. G. WECKeL et H. C. JACKson. L'irradiation du lait. Agricultural Experiment Station of the University of Wisconsin, Madison. Bulletin de Recherches $\mathrm{n}^{\circ} 136$, mars 1939.

\section{NOTE COMPLÉMENTAIRE AU SUJET DE :}

\section{LIRRADIATION DU LATT AUX ÉTATS-UNIS}

Comme nos lecteurs l'auront remarqué, toutes les références que nous avons eitées en fin d'article se réfèrent à la période antérieure à juin 1939. Il semble, en effet, que, depuis cette date, tout au moins aux Etats-Unis, peu d'articles aient été publiés sur cette question, en dehors de ceux dont il est fait mention dans la « Revue des Progrès de la Science laitière. - Section D. - Valeur alimentaire du lait et des produits lactés ". Journal of Dairy Research, vol. 13, no 2, mai 1943, p. 216-141, par S. K. Kon. et qui se réfèrent surtout à la question de la vitamine $D$.

Toutefois, le Pr H.T. Scotr, Directeur des Recherches biologiques de la "Wisconsin Alumni Research Foundation", dans une lettre du 8 janvier 1946, nous indiquait qu'il existe aux Etats-Unis de nombreux travaux non publiés sur l'irradiation qui ont été accumulés pendant ces quatre ou cinq dernières années. Ces travaux ont surtout trait à une modification de la technique de la production du lait irradié pour obtenir un potentiel en vitamine D plus élevé.

Jusqu'à vers 1939, pratiquement tout le lait irradié aux EtatsUnis avait un potentiel de 135 U.S.P. unités de vitamine D par quart $\left(946 \mathrm{~cm}^{3}\right)$. Le Corps médical ayant demandé un potentiel plus élevé en vitamine $\mathrm{D}$, les services du $\mathrm{P}^{\mathrm{r}}$ ScotT étudièrent cette question, et trouvèrent que ceci pouvait être obtenu en utilisant des lampes en quartz à arc de mercure ou à are de carbone plus puissantes. Ils trouvèrent également qu'il était nécessaire de diminuer la vitesse du courant à travers les irradiateurs. Des laits irradiés en bouteilles et des laits frais irradiés contenant 400 unités de vitamine D par quart furent mis sur le marché et jouissent maintenant aux Etats-Unis du plus grand succès de vente.

ScotT et ses collaborateurs trouvèrent également que cette méthode pouvait être appliquée au lait évaporé. Toutefois, lorsqu'ils examinèrent le lait frais et le lait évaporé a u point de vue des autres vitamines que le lait contient, de façon à déterminer si cette super-irradiation eausait une destruction de ces vitamines, ils trouvérent une perte de 8 à $10 \%$ de riboflavine (vitamine $\mathrm{B}^{2}$ ) 
et une destruction de 10 à $12 \%$ de la teneur en vitamine $\mathrm{A}$ du lait. Ceci ne se produit que dans le lait évaporé. Le lait frais, non sujet à l'évaporation, ne subit pas de telles pertes.

Il n'a pas été possible à Scotr et à ses collaborateurs de déterminer là raison de cette différence, quoiqu'ils supposent qu'on puisse l'attribuer à la plus forte viscosité du lait évaporé. En effet, cette viscosité plus élevée tend à ralentir la vitesse du courant du lait et ce dernier est par conséquent exposé plus longtemps à l'effet de l'arc que ne l'est le lait frais en raison de sa viscosité plus faible.

Il résulte de ces observations que le lait évaporé n'est pas irradié aux Etats-Unis.

Il y a environ deux ans, tous les fabricants irradiant le lait évaporé changèrént de méthode : ils ajoutent maintenant un concentré, soit de vitamine $\mathrm{D}^{2}$ de l'ergostérol irradié, soit de vitamine $\mathrm{D}^{3}$ du 7-déshydro-cholestérol et même, dans certains cas, d'extraits d'huile de foie de poisson.

Les mêmes types de concentrés ci-dessus indiqués sont ajoutés dans beaucoup de cas au lait frais.

Bien entendu, une des raisons du passage de l'irradiation directe du lait évaporé à l'addition de concentrés est due au fait que, pendant la guerre, il était impossible d'obtenir l'acier inoxydable qui est nécessaire dans la construction du matériel d'irradiation. Une autre raison était que la place manquait dans la plupart des ateliers de condensation pour installer le matériel d'irradiation supplémentaire nécessaire. Il aurait, en effet, été indispensable pour produire 400 unités d'avoir presque trois fois plus de machines en raison de ce que, plus on désire un potentiel élevé, plus il faut de matériel d'irradiation en raison de la vitesse plus faible du débit du lait.

Le $\mathrm{Pr}^{\mathrm{S}}$ Scotw croit que la faible destruction des vitamines A et $\mathrm{B}^{2}$, mentionnée plus haut, pourrait être évitée. Toutefois, les autres raisons indiquées sont assez importantes pour que les grands ateliers d'évaporation aient été amenés à changer leur méthode d'enrichissement du lait évaporé en vitamine $D$.

Personnellement, nous avons constaté qu'une des plus grandes marques américaines de produits lactés vend maintenant de la poudre de lait complet additionnée de vitamine $D$ en indiquant que 4 onces ( $0 \mathrm{~kg}$. 1134) de lait en poudre produisant un quart (0 1. 946) de lait fluide reconstitué "fournissent 400 unités U.S.P. par jour, e'est-à-dire les exigences journalières complètes n en vitamine D.

Le lait à vitamine $\mathrm{D}$ n'est pas seulement entièrement approuvé - et recommandé depuis plusieurs années par le «Council of Foods and Nutrition of the American Medical Association " (Comité des 
Aliments et de la Nutrition de l'Association médicale américaine), il est largement utilisé et preserit par les médecins américains et, au point de vue commercial, sa qualité a toujours été très convenable, ce qui lui assure une vente des plus régulières.

Le Dr P. C. JE ANs, de l'Ecole de Médecine de l'Université d'Iowa, a indiqué que «les cas de rachitisme aux Etats-Unis sont pratiquement des curiosités médicales et très difficiles à trouver pour démontrer aux différentes classes de l'Ecole de Médecine ce qu'est le rachitisme. ". Ceci illustre les excellents résultats obtenus par l'enrichissement du lait en vitamine D. Le Dr J. M. Lewis, de New-York City, a fait une déclaration analogue à celle du Dr JEANS.

Avec du lait à vitamine $\mathrm{D}$ on possède une méthode automatique de prévention du rachitisme, et le Pr Scotw conclut que la nécessité de fournir de la vitamine $D$ est aussi bien admise que celle de fournir le lait lui-même et que la combinaison de la vitamine $D$ au lait rend impossible l'omission de cette vitamine dont le petit enfant et l'enfant en croissance ont un besoin tellement impérieux.

\title{
ANALYSE DES CRÈMES
}

\section{Modification du Modus opérandi Koehler-Bacot}

\author{
par
}

\section{RAPHAEL MOREAU}

La modifieation, objet de la présente note, a pour but l'amélioration des conditions de lecture.

Dans la méthode Kœhler-Bacot, après introduction dans le butyromètre d'un poids de crème voisin de 5 grammes, les réactifs sont ajoutés dans l'ordre décroissant des densités : acide sulfurique, ean, alcool amylique.

Lors de l'agitation, le mélange de l'acide sulfurique à l'eau provoque une élévation de température et détermine une carbonisation des matières organiques de la crème. Cette carbonisation se traduit par une coloration anormale de la matière grasse qui prend une teinte voisine de celle prise par les matières protéiques et les autres matières organiques contenues dans la crème.

Cette coloration dépend d'ailleurs de la richesse de la crème, de sa viscosité, de son acidité et des impuretés contenues. La lecture est de ce fait souvent difficile voire même parfois impossible car il est très rare que la matière grasse ne soit pas-attaquée.

Une a mélioration est constatée si, conformément au processus "Kœhler ", l'acide sulfurique est d'abord introduit dans le butyromètre, mais la manipulation de ce dernier n'est pas alors sans 